\title{
Extraspinal osteoarticular multidrug-resistant tuberculosis in children: A case series
}

\author{
G B Firth, ${ }^{1}$ MB BCh, FCS (Orth), MMed (Orth); J Lescheid, ${ }^{1}$ BSc, MD, FRCSC; M Camacho, ${ }^{1}$ MB BCh; M Esteves, ${ }^{1}$ BCMP; \\ N Beylis, ${ }^{2}$ MB BCh; M J Groome, ${ }^{3,4}$ MB BCh, MSc, PhD; S A Madhi, ${ }^{3,4}$ MB BCh, FCPaeds (SA), PhD

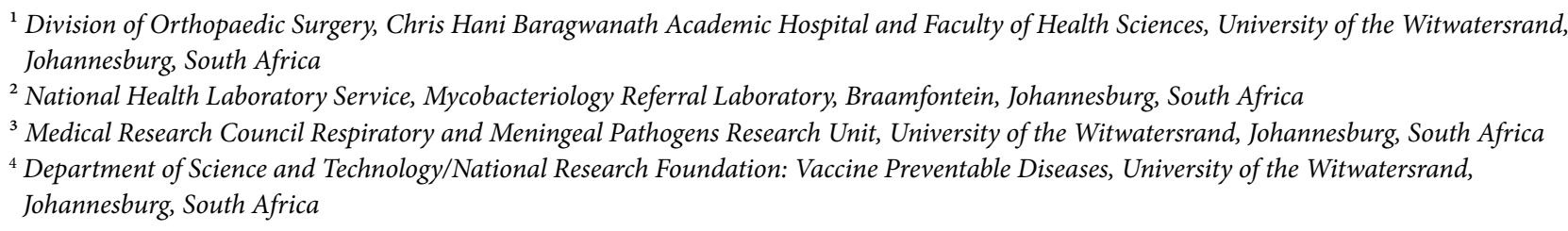

Corresponding author: $M J$ Groome (groomem@rmpru.co.za)

\begin{abstract}
Background. South Africa (SA) is known to have a high disease burden of tuberculosis (TB). Extraspinal osteoarticular multidrug-resistant tuberculosis (ESOA MDR-TB) in children has only been described in a few case reports worldwide.

Objectives. To describe the epidemiology and highlight the potential problem of ESOA MDR-TB infections as seen in children from a single academic hospital in SA.

Methods. A retrospective record review was performed on all children diagnosed with ESOA TB infection at Chris Hani Baragwanath Academic Hospital, Johannesburg, between 1 January 2006 and 31 December 2015. All patients with a positive TB culture (fluid or tissue) from the surgical site of biopsy (bone or joint) and who were hospitalised were included. Organism culture and drug sensitivity testing were performed.

Results. Overall 19 cases of ESOA TB were identified. Areas involved included the shoulder ( 2 cases), elbow ( 2 cases), hip ( 7 cases), knee (4 cases), ankle (3 cases) and humerus (1 case). The mean age of the population was 7.7 (range 2.0 - 14.0) years. The mean white cell count was 11.3 (range 5 - 28.9) $\times 10^{9} / \mathrm{L}$, the mean C-reactive protein level 53.8 (range 1.0 - 364.0) $\mathrm{mg} / \mathrm{L}$ and the mean erythrocyte sedimentation rate 35.5 (range $4.0-85.0) \mathrm{mm} / \mathrm{h}$. Two cases $(10.5 \%$ ) were MDR, and a further case (5.3\%) was resistant to isoniazid only. Four of 12 patients tested positive for HIV. One of the HIV-positive patients was isoniazid resistant. The two positive ESOA MDR-TB cases are discussed in detail.

Conclusions. These findings indicate that ESOA MDR-TB is a reality in this paediatric population (10.5\%) and a high index of suspicion should be maintained, especially when cultures are negative in children with signs and symptoms of ESOA TB. The effect of HIV infection on the incidence of ESOA MDR-TB requires further study.
\end{abstract}

S Afr Med J 2017;107(11):983-986. DOI:10.7196/SAMJ.2017.v107i11.12577

The burden of tuberculosis (TB) worldwide is significant, causing an estimated $5 \%$ of global paediatric deaths annually. ${ }^{[1]}$ South Africa (SA) is known to have a high burden of disease, ${ }^{[2]}$ with children accounting for $10-20 \%$ of all TB cases. Less than $2 \%$ of new cases in SA are due to multidrug-resistant (MDR)-TB. ${ }^{[1,3]}$ Extraspinal osteoarticular (ESOA) TB comprises only 2 - 3\% of all TB cases. ${ }^{[4]}$ Children are more likely than adults to contract ESOA TB owing to increased bacterial adherence and venous stasis in tortuous metaphyseal vessels. Diagnosis can be difficult with paucibacillary disease, indolent presentation, nonspecific blood and imaging findings, and adequate tissue samples being difficult to obtain. ${ }^{[1,4,5]}$ Early diagnosis and effective treatment are usually critical for full recovery, since the results of non-treatment or delays in treatment can be debilitating. ${ }^{[1,5-7]}$

MDR-TB is defined as Mycobacterium tuberculosis that is resistant to both isoniazid (INH) and rifampicin (RIF), regardless of other susceptibilities. ${ }^{[1]}$ Exposure to MDR-TB is the primary mode of spread, so early detection is imperative to limit spread. In SA, MDRTB has been declared an epidemic in its major centres. ${ }^{[8,9]}$ There is no literature that explores ESOA MDR-TB in children.

\section{Objectives}

To describe the epidemiology and highlight the potential problem of ESOA MDR-TB infections as seen in children from a single academic hospital in SA.

\section{Methods}

A retrospective chart review of children diagnosed with ESOA TB was performed. All the patients were hospitalised at Chris Hani Baragwanath Academic Hospital, Soweto, Johannesburg, between 1 January 2006 and 31 December 2015. The National Health Laboratory Service database was used to access results. The diagnosis of TB was solely based on the child having a positive TB culture (acid-fast bacilli (AFB) taken from the affected site (fluid or tissue)). Organism culture was performed using the Mycobacterial Growth Indicator Tube (MGIT 960) method, and drug sensitivity testing was done. All children aged $\leq 14$ years with ESOA TB with positive TB culture results were included in the study. All other children who were diagnosed with ESOA TB on clinical grounds and treated with the standard protocol for ESOA TB were excluded, as the main aim of the study was to review children with ESOA MDR-TB. Polymerase chain reaction (PCR) testing was not done routinely in the study and therefore could not be used for assessment.

The following blood results were recorded: white cell count (WCC), C-reactive protein (CRP) level, erythrocyte sedimentation rate (ESR) and HIV enzyme-linked immunosorbent assay (ELISA). All histology specimens that were taken were recorded, specifically looking for chronic granulomatous inflammation and the presence of Langerhans multinucleated giant cells to confirm the diagnosis of TB. 


\section{Ethical approval}

Ethics clearance was obtained from the University of the Witwatersrand (ref. no. M140259).

\section{Results}

Over the 10-year study period there were 19 cases of culture-positive ESOA TB. Sixteen of the cases were sensitive to all drugs. Two cases (10.5\%) were MDR, and neither tested positive for HIV. One case was resistant to INH, and this patient was HIV-positive. The mean age for the total group was 7.7 (range $2.0-14.0$ ) years. The mean WCC was 11.3 (range 5 - 28.9) $\times 10^{9} / \mathrm{L}$, the mean CRP level was 53.8 (range 1.0 $364.0) \mathrm{mg} / \mathrm{L}$, and the mean erythrocyte sedimentation rate (ESR) was 35.5 (range $4.0-85.0$ ) $\mathrm{mm} / \mathrm{h}$. Twelve patients were tested for HIV, 4 of whom were HIV-positive (33.3\%). Histological examination was performed on 12 specimens, and 10 confirmed features in keeping with TB, namely chronic granulomatous inflammation.

\section{The MDR cases}

Case 1

A 2-year-old girl, who had previously been well, presented with a 2-day history of swelling, tenderness and decreased movement of the right arm. On examination she was febrile and had a warm, swollen right shoulder and elbow with associated pseudoparalysis of both joints. She had leucocytosis (WCC $19.85 \times 10^{9} / \mathrm{L}$ ) with a low haemoglobin concentration $(10.6 \mathrm{~g} / \mathrm{dL})$. A diagnosis of acute bacterial osteoarticular infection was made and intravenous ceftriaxone therapy was initiated. She was then taken as an emergency to the operating theatre for arthrotomy of the right shoulder and elbow joints. A large amount of purulent material was evacuated from both joints, and a thorough washout was performed. Swabs, fluid and tissue samples were sent for standard bacterial and mycobacterial culture and sensitivity, and tissue samples were sent for histological examination. Postoperatively the leucocytosis resolved by day 2, but the ESR and CRP remained elevated at $133 \mathrm{~mm} / \mathrm{h}$ and $367.8 \mathrm{mg} / \mathrm{L}$, respectively. The antibiotic was changed to intravenous cloxacillin based on a pus culture result of sensitive Staphylococcus aureus. Blood cultures were negative for bacterial growth and pus samples were negative for AFB. The histological findings were remarkable for synovitis with an inflammatory infiltrate and bacterial colonies, but no granulomatous changes, and negative Ziehl-Neelsen and periodic acid-Schiff (PAS) stains. A relook arthrotomy after 3 days was negative.

Clinical recovery was complete after 2 weeks of intravenous cloxacillin, followed by 4 weeks of oral cloxacillin. After 6 weeks, mycobacterial culture and sensitivity results from the right shoulder tissue biopsy specimen revealed $M$. tuberculosis resistant to INH and RIF. Owing to a fully resolved clinical picture, negative CRP $(1.0 \mathrm{mg} / \mathrm{L})$, a mildly elevated ESR $(50 \mathrm{~mm} / \mathrm{h})$, negative HIV ELISA and negative blood and sputum TB cultures, it was decided not to administer anti-TB treatment. At follow-up after 1 year, the patient had close to full range of movement in the shoulder and elbow joints and the ESR was $22 \mathrm{~mm} / \mathrm{h}$. Radiographs of the right humerus and elbow joint showed no active disease, but areas of healed infective changes were visible.

\section{Case 2}

A 12-year-old boy on chronic prednisone and methotrexate treatment for resistant juvenile dermatomyositis was referred with a residual fixed-flexion contracture of his right knee. The dermatomyositis was difficult to control, with recurrent ulcerations and vasculitic rashes despite ongoing methotrexate and prednisone treatment. The previous history revealed that at the age of 6 years he had developed a spontaneous abscess just distal to the right knee, which cultured vancomycin-sensitive methicillin-resistant $S$. aureus. This resolved completely after surgical drainage and 2 weeks of intravenous vancomycin and further oral clindamycin. During his 7 th year, he developed septic arthritis of the right knee and elbow, which cultured methicillin-sensitive $S$. aureus and was treated with another surgical drainage and cloxacillin for 6 weeks. At 7 years of age, a BACTEC culture was positive for $M$. tuberculosis after he presented with recurrent swelling and draining sinuses from his right knee and wrist. Standard anti-TB therapy was initiated and significant clinical improvement of affected joints was noted after 1 month. One month later, he returned with a recurrent abscess in his right leg, with a positive tissue culture for INH-resistant, RIF-sensitive TB. Therapy was reinitiated with RIF, INH, ethionamide (ETA) and pyrazinamide (PZA). Again clinical improvement was noted, and after a finding of mildly elevated transaminases after 17 months of treatment, PZA was removed from the therapeutic regimen. Treatment was discontinued after a total of 20 months. Methotrexate was discontinued after the initial diagnosis of TB and restarted 2 months after the final dose of triple therapy for the monoresistant $\mathrm{TB}$.

On the patient's most recent admission, the knee had multiple surgical scars from previous arthrotomies for bacterial septic arthritis and ankylosis at $80^{\circ}$ of flexion, and was painful (Fig. 1). Pain and globally limited range of motion were also evident in the right hip. There were no draining sinuses. Radiographs showed a periosteal reaction in the mid-shaft of the right femur, symmetrical loss of joint space in the knee with osteopenia, preservation of the physes and no bony erosions (Fig. 2). Radiographs of the right hip were remarkable for complete loss of joint space with extensive femoral and acetabular bone loss (Fig. 3). The WCC was normal at $7.89 \times 10^{9} / \mathrm{L}$, but the ESR

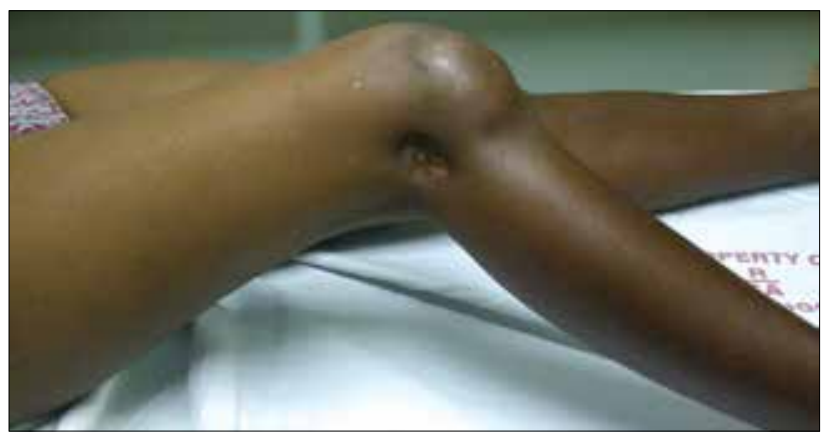

Fig. 1. Case 2. Clinical picture of knee flexion contracture and scarred sinuses.

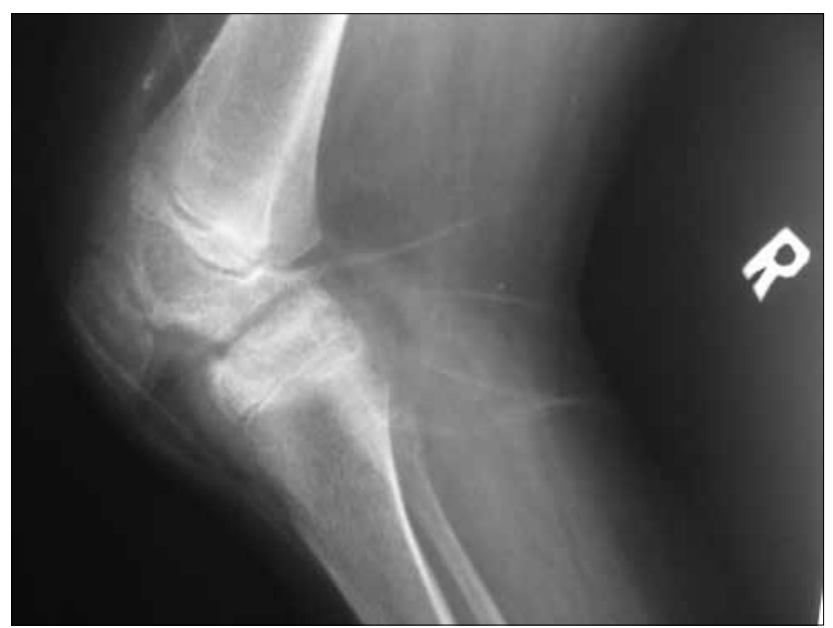

Fig. 2. Case 2. Lateral radiograph of the knee showing destruction of joint. 


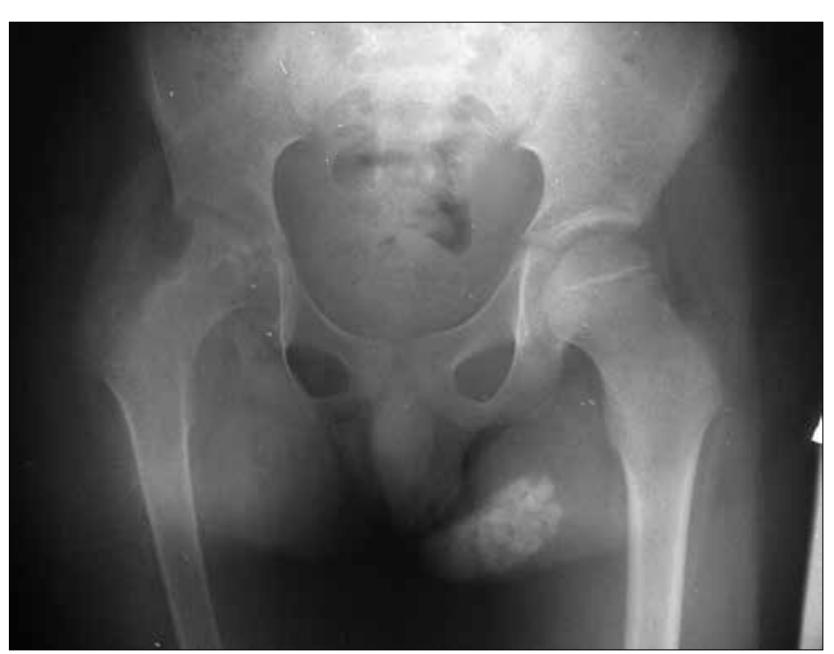

Fig. 3. Case 2. Anteroposterior radiograph of the pelvis showing destruction of the right hip joint with erosions of the femoral head and joint space narrowing.

was elevated at $40 \mathrm{~mm} / \mathrm{h}$. He was taken to the operating theatre for manipulation of the right knee and biopsy of the right hip. Purulent material and tissue were evacuated from the hip and sent for histology, microscopy, and bacterial and mycobacterial culture and sensitivity testing. Histological findings were of extensive necrotising granulomatous inflammation, positive Ziehl-Neelsen staining and a negative PAS stain. PCR testing of the specimen was performed after a positive TB culture, and revealed resistance to INH and RIF. Treatment with streptomycin, amikacin and ciprofloxacin was given for a total of 24 months. At last clinical follow-up, his hip and knee remained ankylosed but were no longer painful or inflamed.

\section{Discussion}

The study presents 19 paediatric ESOA TB culture-positive cases over 10 years. Two cases of MDR-TB comprised 10.5\% of the total of 19 cases seen. One case (5.3\%) was INH resistant only. ESOA MDR-TB is rarely reported, especially in children, and these two cases add to the extremely sparse literature on paediatric ESOA MDR-TB. Previous publications are limited to case reports and subanalyses of populations consisting of adults and children with pulmonary MDRTB. ${ }^{[10-12]}$ A series from Cape Town, SA, potentially represents the largest case series of paediatric MDR-TB to date. Among 238 children with clinically diagnosed TB, 6 cases were osteoarticular, and 8 cases overall were identified as MDR-TB. Unfortunately, it is not clear how many of the MDR-TB cases were osteoarticular. ${ }^{[3]}$ Of 106 cases of paediatric osteoarticular TB over 20 years prior to 2001 in Rabat, Morocco, none were identified as drug resistant, and all 80 patients who were not lost to follow-up reportedly recovered fully after firstline therapy. ${ }^{[13]}$ This study is the largest review of ESOA MDR-TB to date in the English literature.

Spontaneous remission of ESOA MDR-TB, as in case 1, has not been recorded previously. This is the first known case of clinical resolution of symptoms in a joint with proven bacterial septic arthritis and concomitant MDR-TB infection without TB treatment. Potential possibilities are a latent infection in the face of bacterial superinfection, actual spontaneous clearance of the pathogen, or an incorrectly labelled specimen. To the authors' knowledge, there are no recent reports of spontaneous remission even of sensitive TB, although in the pre-antibiotic era, $\sim 50 \%$ of those with active disease did survive at least into clinical cure. ${ }^{[14]}$ Only two studies address the possibility of spontaneous cure. Winqvist et al. ${ }^{[15]}$ found that current rates of TB in Sweden are far lower than would be expected given the estimated incidence of infection in the pre-antibiotic era, and reactivation from age-related immunocompromise. Their conclusion was that some probands were actually eradicating the mycobacterium without the assistance of antibiotics. ${ }^{[15]}$ When examining the cellular immunity profiles of 27 survivors of active TB who never received antimycobacterial chemotherapy, Vynnycky and Fine ${ }^{[14]}$ found seven patients with antimycobacterial-appropriate central memory $\mathrm{T}$ cells in the absence of effector memory $\mathrm{T}$ cells, indicating proof of previous active infection in the absence of any persisting antigen. ${ }^{[16]}$ These articles, although interesting, have not changed the authors' or current World Health Organization (WHO) management guidelines for the treatment of ESOA MDR-TB. ${ }^{[1]}$

The relationship of immunity to MDR-TB has been established. ${ }^{[3,11]}$ Case 2 had immunocompromise due to dermatomyositis and its treatment, and the isoniazid-resistant patient was HIVpositive. Although there have been no specific investigations of the relationships between immunocompromise and development of ESOA MDR-TB in any population, the immunocompromised state has been associated with an increased prevalence of pulmonary MDR-TB ${ }^{[17]}$ and increased mortality from MDR-TB. ${ }^{[18]}$ Drobniewski et $a l .{ }^{[18]}$ found a nine times greater relative risk of mortality in immunocompromised compared with non-immunocompromised patients, with a significant reduction of risk if appropriate treatment was initiated rapidly. In the current study, HIV testing in 12 patients resulted in 4 positive cases (33.3\%). Neither of the ESOA MDR-TB cases was HIV-positive. Given the current prevalence of HIV in the study population, there appears to be no strong relationship between HIV infection and ESOA MDR-TB. This has been corroborated by studies including the same study population. ${ }^{[3,7,10-13,19,20]}$

Both cases of osteoarticular MDR-TB had a history of concomitant bacterial infection in the same site at which the TB was found. No other studies on ESOA MDR-TB have delineated a relationship between TB and bacterial co-infection. However, it has been shown in human immunological studies ${ }^{[21]}$ that infection with helminths decreases cellular immunity to TB. In a mouse model of malaria-associated acute respiratory distress syndrome, co-infection with Plasmodium berghei caused a significant exacerbation of $\mathrm{TB}$ infection. ${ }^{[22]}$ Furthermore, antibody titres against influenza A/ $\mathrm{H} 3 \mathrm{~N} 2$ were higher in a cohort of Indonesians with new clinical manifestations of TB than in controls, and highest in those with the most severe manifestations of TB. ${ }^{[23]}$ Since viral and parasitic infections increase susceptibility to TB activation/infection, it is plausible that bacterial infection may have the same negative immune-modulatory effect in children with ESOA MDR-TB. It is certainly interesting to think that treatment of a bacterial infection in case 1 may have resulted in cure or latency of the TB co-infection.

Standard drug susceptibility testing requires a positive culture, which is only present in $\sim 40 \%$ of cases ${ }^{[5,7,24,25]}$ and gives results at an average of 36 - 45 days after sample collection. ${ }^{[7]}$ Many molecularbased tests have been developed to improve the detection and susceptibility of MDR-TB, but unfortunately were not available for many of the patients in this cohort. ${ }^{[25-27]}$ Use of the GenoType MTBDRplus assay has been shown to reduce time to initiation of appropriate drug therapy by 25 days compared with BACTEC MGIT 960 in rural SA. ${ }^{[28]}$ Many new diagnostic technologies have been described in the most recent report on TB by the WHO to improve lag time to diagnosis and sensitivity testing for appropriate treatment of MDR-TB. ${ }^{[1]}$

Donald ${ }^{[24]}$ recommended the following treatment in children with osteoarticular TB: 2 months of triple-therapy intensive phase with INH 5 - $15 \mathrm{mg} / \mathrm{kg} / \mathrm{d}$, RIF $10-20 \mathrm{mg} / \mathrm{kg} / \mathrm{d}$ and PZA $30-40 \mathrm{mg} /$ 
$\mathrm{kg} / \mathrm{d}$, followed by 4 months of double-therapy continuation phase with INH and RMP. In cases where relapse would have especially disastrous consequences (spinal TB) or if it would go unnoticed, the continuation phase should extend for 12 months. If there is any suspicion of drug resistance, ETA $15-25 \mathrm{mg} / \mathrm{kg} / \mathrm{d}$ should be added for all phases. Seddon et al ${ }^{[19]}$ treated seven children with cultureconfirmed spinal MDR-TB in Cape Town, SA, between 2004 and 2010 using the WHO general guidelines (treatment of at least 18 months' duration with at least four drugs to which the organism is susceptible, incorporating an initial 6-month intensive phase using at least one injectable drug). They reported that all six surviving children at the end of the study had tolerated the treatment well with positive clinical outcomes and fewer side-effects than expected. Adding to these recommendations, Al-Dabbagh et al ${ }^{[5]}$ stressed that three of the selected drugs should not have been administered before, two should be bactericidal, and if possible, either an injectable aminoglycoside or fluoroquinolone should be used during the intensive phase. They also placed the appropriate multidrug regimen within an effective treatment plan involving reliable drug susceptibility testing, directly observed therapy by a specialised team to ensure adherence, and close monitoring and management of expected side-effects specific to the drugs. ${ }^{[1,5]}$

Surgery for diagnosis of ESOA TB is essential, and with the high rate of bacterial co-infection as seen in this study, drainage, debridement and washout of the joint is important. Surgery played an important role in both the cases presented above. Traditionally, spinal $\mathrm{TB}$ has been treated non-surgically because of excellent results with non-surgical treatment, extending even to resolution of erosions, and improvement in vertebral height. ${ }^{[29]}$ Surgical indications include abscesses causing neurological compromise, septic dislocation and treatment of residual instability or deformity secondary to bony destruction or growth disturbance. ${ }^{[10,12,13]}$ In the ESOA MDR-TB literature, many have observed that surgical treatment appears to improve outcome, but no formal statistical analysis has been performed. ${ }^{[19,29]}$ In contrast, it is well established in the pulmonary literature on MDR-TB that surgery improves the chance of a favourable outcome. ${ }^{[30]}$

\section{Study limitations}

The study had a number of limitations. It was retrospective, the focus being to look at the prevalence of ESOA MDR-TB in children at a tertiary institution, which may have skewed the sample towards being less representative of the general population. The number of cases seen at a regional hospital should be minimal, however, as all paediatric orthopaedic pathology is referred to the study hospital. The positive TB cultures required for inclusion of the study population meant that a potentially large number of patients who were treated for ESOA TB based on clinical grounds were excluded.

\section{Conclusions}

There was a relatively high prevalence of paediatric ESOA MDR-TB $(10.5 \%)$ in this population. This finding should stimulate vigilance with regard to rapid and reliable early means of detection with newer genetic tests and drug susceptibility testing in all cases, with knowledge of and adherence to published specific treatment guidelines. Appropriate chemotherapy should be rapidly initiated, aggressive and prolonged, and adjunctive surgery is always indicated for initial debridement and diagnostic sampling.

\section{Acknowledgements. None.}

Author contributions. All the authors made substantial contributions to conceptualisation and design of the study, analysis and interpretation of data, and drafting and critical revision of the article.

Funding. None.

Conflicts of interest. None.

1. World Health Organization. Global tuberculosis report 2014. http://apps.who.int/iris/ bitstream/10665/137094/1/9789241564809_eng.pdf (accessed 30 September 2017).

2. Glaziou P, Falzon D, Floyd K, Raviglione M. Global epidemiology of tuberculosis. Semin Respir Crit Care Med 2013;34(1):3-16. https://doi.org/10.1101/cshperspect.a017798

3. Soeters M, de Vries AM, Kimpen JL, Donald PR, Schaaf HS. Clinical features and outcome in children admitted to a TB hospital in the Western Cape - the influence of HIV infection and drug resistance. S Afr Med J 2005;95(8):602-606.

4. Ludwig B, Lazarus AA. Musculoskeletal tuberculosis. Dis Mon 2007;53(1):39-45. https://doi, org/10.1016/.jisamonth.2006.10.005

5l-Dabbagh M, Lapphra K, McGloin R, et al. Drug resistant TB: Pediatric guidelines. Pediatr Infect Dis I 2011;30(6):501-505. https://doi.org/10.1097/INF.0b013e31820b583d

6. Campbell JA, Hoffman EB. Tuberculosis of the hip in children. J Bone Joint Surg Br 1995;77(2):319-326.

7. Li L, Zhang Z, Luo F, et al. Management of drug-resistant spinal tuberculosis with a combination of Li L, Zhang Z, Luo F, et al. Management of drug-resistant spinal tuberculosis with a combination of
surgery and individualised chemotherapy: A retrospective analysis of thirty-five patients. Int Orthop surgery and individualised chemotherapy: A retrospective anal/sis

8. Marais BJ, Mlambo CK, Rastogi N, et al. Epidemic spread of multidrug-resistant tuberculosis in 8. Marais BJ, Mlambo CK, Rastogi N, et al. Epidemic spread of multidrug-resistant tuberculosis in
Johannesburg, South Africa. J Clin Microbiol 2013;51(6):1818-1825. https://doi.org/10.1128/JCM.00200-13 9. Seddon JA, Hesseling AC, Marais BJ, Jordaan A, Victor T, Schaaf HS. The evolving epidemic of drugresistant tuberculosis among children in Cape Town, South Africa. Int J Tuberc Lung Dis 2012;16(7):928933. https://doi.org/10.5588/ijtld.11.0679

10. Rodriguez L, Weinberg B, Benaroya A, Gertner M. Multidrug-resistant tuberculous osteomyelitis. Pediatr Infect Dis J 1997;16(11):1096. https://doi.org/10.1097/00006454-199711000-00022

11. Lindquist SW, Steinmetz BA, Starke JR. Multidrug-resistant tuberculosis of the first cervical vertebra in an immunocompetent adolescent. Pediatr Infect Dis J 1997;16(3):333-336. https://doi. org/10.1097/00006454-199703000-00020

12. Baquero-Artigao F, Garcia-Miguel MJ, Merino R, García-Consuegra J, del Castillo F. Multidrugresistant tuberculosis of the ankle: Case report. Foot Ankle Int 2006;27(8):642-644. https://doi. $\mathrm{org} / 10.1177 / 107110070602700815$

13. Teklali Y, El Alami ZF, El Madhi T, Gourinda H, Miri A. Peripheral osteoarticular tuberculosis in children: 106 case-reports. Joint Bone Spine 2003;70(4):282-286. https://doi.org/10.1016/S1297-319X(03)00037-X

4. Vynnycky E, Fine PE. The annual risk of infection with Mycobacterium tuberculosis in England and Wales since 1901. Int J Tuberc Lung Dis 1997;1(5):389-396.

15. Winqvist N, Björk J, Miörner H, Björkman P. Long-term course of Mycobacterium tuberculosis infection in Swedish birth cohorts during the twentieth century. Int J Tuberc Lung Dis 2011;15(6):736-740. https:// doi.org/10.5588/ijtld.10.0683

16. Millington KA, Gooding S, Hinks TSC, Reynolds DJM, Lalvani A. Mycobacterium tuberculosis-specific cellular immune profiles suggest bacillary persistence decades after spontaneous cure in untreated tuberculosis. J Infect Dis 2010;202(11):1685-1689. https://doi.org/10.1086/656772

17. Jiang JR, Yen SY, Wang JY. Increased prevalence of primary drug-resistant pulmonary tuberculosis in immunocompromised patients. Respirology 2011;16(2):308-313. https://doi.org/10.1111/j.14401843.2010.01902.x

18. Drobniewski F, Eltringham I, Graham C, Magee JG, Smith EG, Watt B. A national study of clinical and laboratory factors affecting the survival of patients with multiple drug resistant tuberculosis in the UK. Thorax 2002;57(9):810-8116. https://doi.org/10.1136/thorax.57.9.810

19. Seddon JA, Donald PR, Vlok GJ, Schaaf HS. Multidrug-resistant tuberculosis of the spine in children . Seddon JA, Donald PR, Vlok GJ, Schaaf HS. Multidrug-resistant tuberculosis of the spine in children -
characteristics from a high burden setting. I Trop Pediatr 2012;58(5):341-347. https://doi.org/10.1093/ tropej/fmrl04

20. Fairlie L, Beylis NC, Reubenson G, Moore DP, Madhi SA. High prevalence of multi-drug resistance in tuberculosis in Johannesburg, South Africa: A cross sectional study. BMC Infect Dis 2011;11:28. https:// doi.org/10.1186/1471-2334-11-28

21. Rafi W, Ribeiro-Rodrigues R, Ellner JJ, Salgame P. Coinfection-helminthes and tuberculosis. Curr Opin HIV AIDS 2012;7(3):239-244. https://doi.org/10.1097/COH.0b013e3283524dc5

22. Mueller AK, Behrends J, Hagens K, Mahlo J, Schaible UE, Schneider BE. Natural transmission of Plasmodium berghei exacerbates chronic tuberculosis in an experimental co-infection model. PLoS One 2012;7(10):e48110. https://doi.org/10.1371/journal.pone.0048110

23. De Paus RA, van Crevel R, van Beek R, et al. The influence of influenza virus infections on the development of tuberculosis. Tuberculosis 2013;93(3):338-342. https://doi.org/10.1016/j.tube.2013.02.006

24. Donald PR. The chemotherapy of osteo-articular tuberculosis with recommendations for treatment of children. J Infect 2011;62(6):411-439. https://doi.org/10.1016/j.jinf.2011.04.239

25. Zhang Z, Li L, Luo F, et al. Rapid and accurate detection of RMP- and INH-resistant Mycobacterium Zhang Z, Li L, Luo F, et al. Rapid and accurate detection of RMP- and INH-resistant Mycobacterium
tuberculosis in spinal tuberculosis specimens by CapitalBioTM DNA microarray: A prospective tuberculosis in spinal tuberculosis specimens by CapitalBioTM DNA microarray: A
validation study. BMC Infect Dis 2012;12:303. https://doi.org/10.1186/1471-2334-12-303

26. Chang K, Lu W, Wang J, et al. Rapid and effective diagnosis of tuberculosis and rifampicin resistance with Xpert MTB/RIF assay: A meta-analysis. J Infect 2012;64(6):580-588. https://doi.org/10.1016/j. jinf.2012.02.012

27. Hanrahan CF, Dorman SE, Erasmus L, et al. The impact of expanded testing for multidrug resistant tuberculosis using genotype MTBDRplus in South Africa: An observational cohort study. PLoS One 2012;7(11):e49898. https://doi.org/10.1371/journal.pone.0049898

28. Jacobson KR, Theron D, Kendall EA, et al. Implementation of GenoType MTBDRplus reduces time to multidrug-resistant tuberculosis therapy initiation in South Africa. Clin Infect Dis 2013;56(4):503-508. https://doi.org/10.1093/cid/cis920

29. Li L, Zhang Z, Luo F, et al. Management of drug-resistant spinal tuberculosis with a combination of surgery and individualised chemotherapy: A retrospective analysis of thirty-five patients. Int Orthop 2012;36(2):277-283. https://doi.org/10.1007/s00264-011-1398-0

30. Gegia M, Kalandadze I, Kempker RR, Magee MJ, Blumberg HM. Adjunctive surgery improves treatment outcomes among patients with multidrug-resistant and extensively drug-resistant tuberculosis. Int $J$ Infect Dis 2012;16(5):e391-e396. https://doi.org/10.1016/j.jiji.2011.12.018 